\title{
Determination and Correlation of Moxidectin Solubility in Aqueous Cosolvents of Methanol, Ethanol, Isopropanol and Ethylene Glycol
}

Chunjuan Huang*, Renjie Xu

Guangling College, Yangzhou University, Yangzhou, Jiangsu 225000, People's Republic of China

Corresponding author. Phone: +86514 87993918; Fax: + 8651487994009.

E-mail address: HCJtzk1978@163.com 

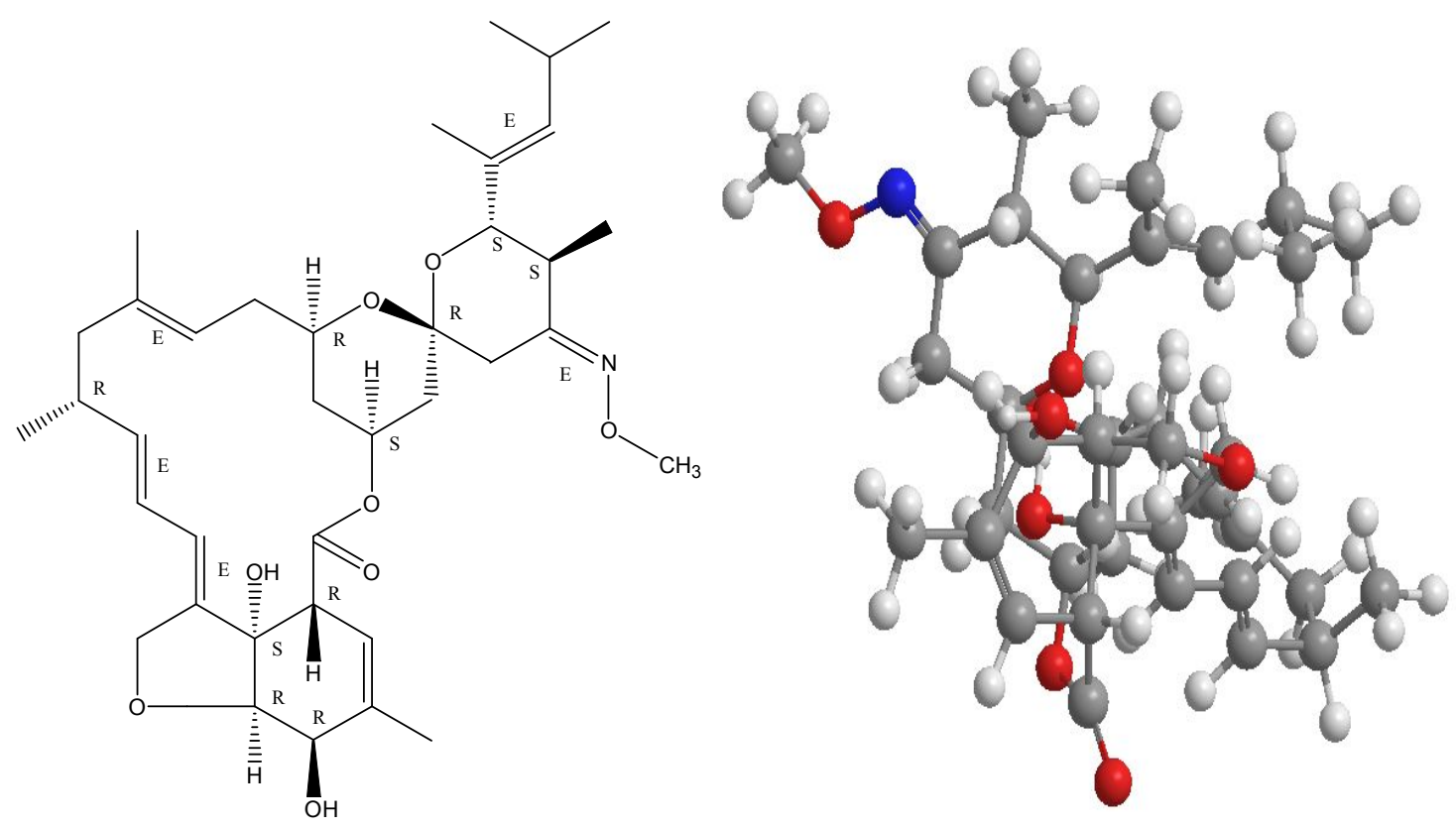

Fig S1. Molecular structure of moxidectin 


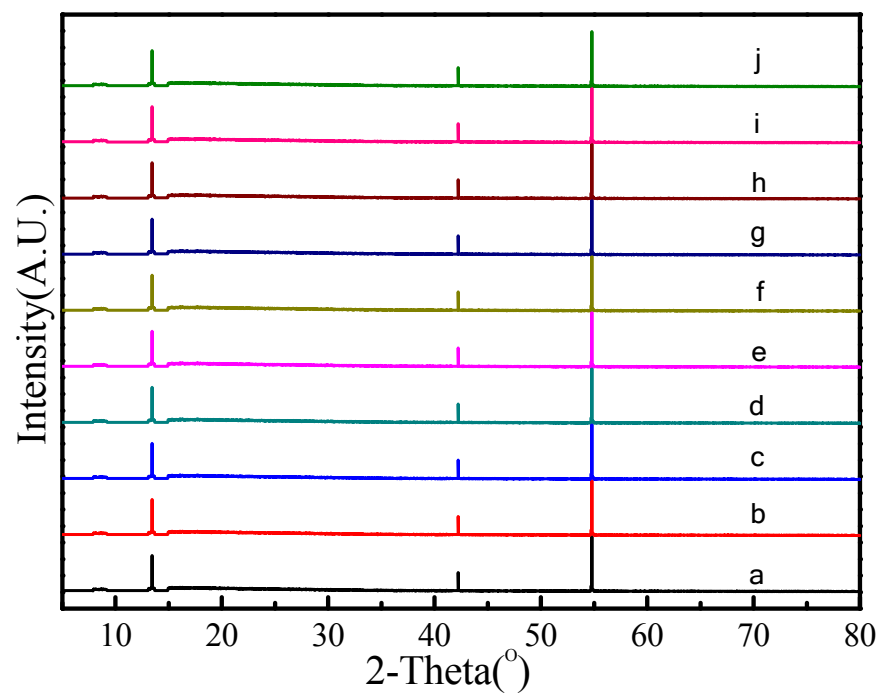

Fig. S2. XPRD spectrogram patterns of moxidectin in pure solvents and different cosolvency systems compared with the raw material. (a) raw material; (b) crystallized in methanol; (c) crystallized in ethanol (d) crystallized in isopropanol; (e) crystallized in EG; (f) crystallized in water; (g) crystallized in methanol + water mixture; (h) crystallized in ethanol + water mixture; (i) crystallized in isopropanol + water mixture; (j) crystallized in EG + water mixture. 

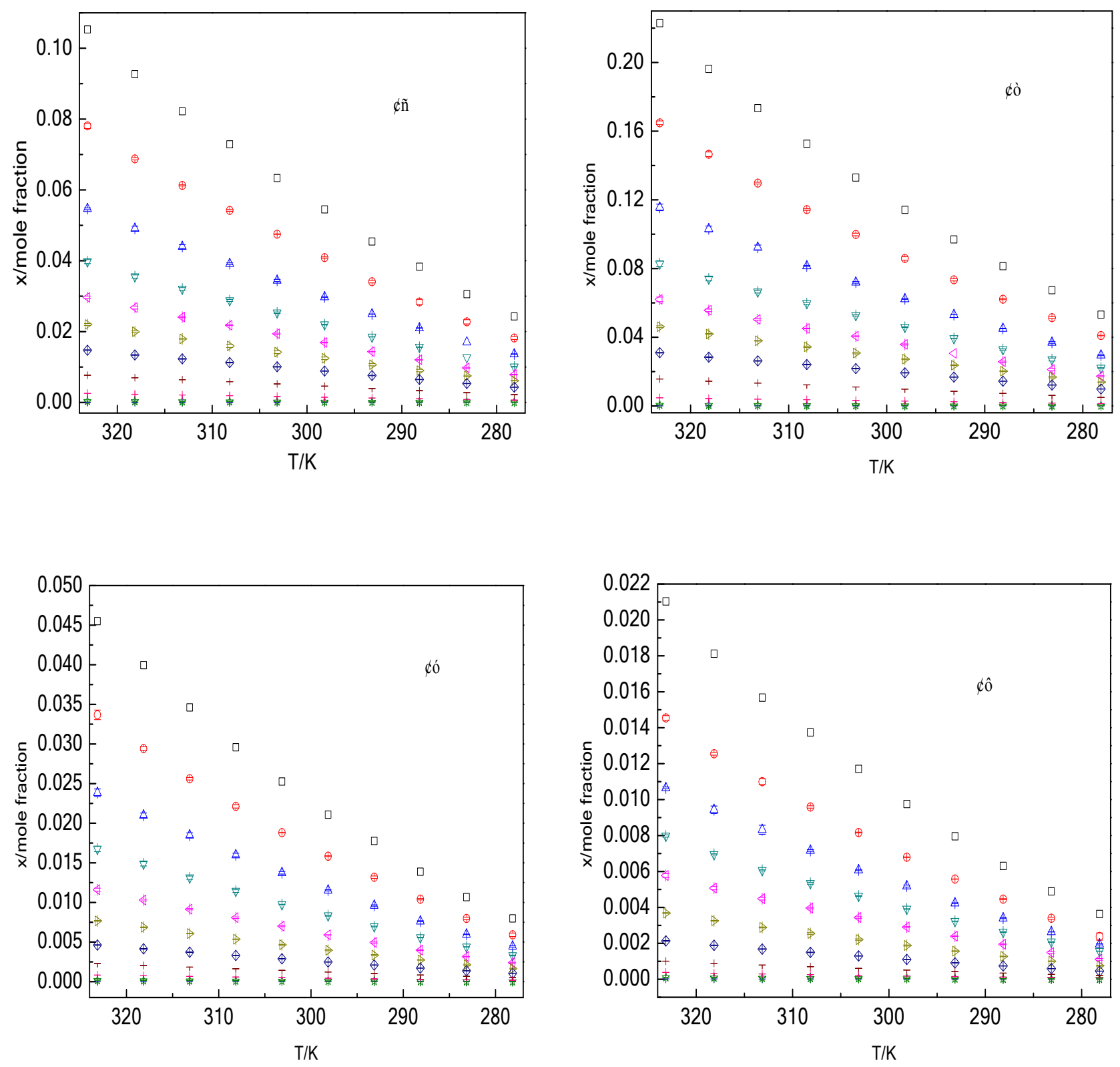

Fig S3. The deviation plot between moxidectin solubility $(x)$ and calculated value of Jouyban-Acree model in four mixtures with various mass fractions at different temperatures: w, mass fraction of methanol ( I ); ethanol (II); isopropanol (III) and EG (IV); $\square, \mathrm{w}=1 ; \bigcirc, \mathrm{w}=0.9 ; \triangle, \mathrm{w}=0.8 ; \nabla, \mathrm{w}=0.7 ; \triangleleft, \mathrm{w}=0.6 ; \triangleright, \mathrm{w}=0.5 ; \diamond, \mathrm{w}=0.4 ; \square-, \mathrm{w}=0.3 ; \mid$, $\mathrm{w}=0.2 ;$ 岤, $\mathrm{w}=0.1 ;+, \mathrm{w}=0$. 

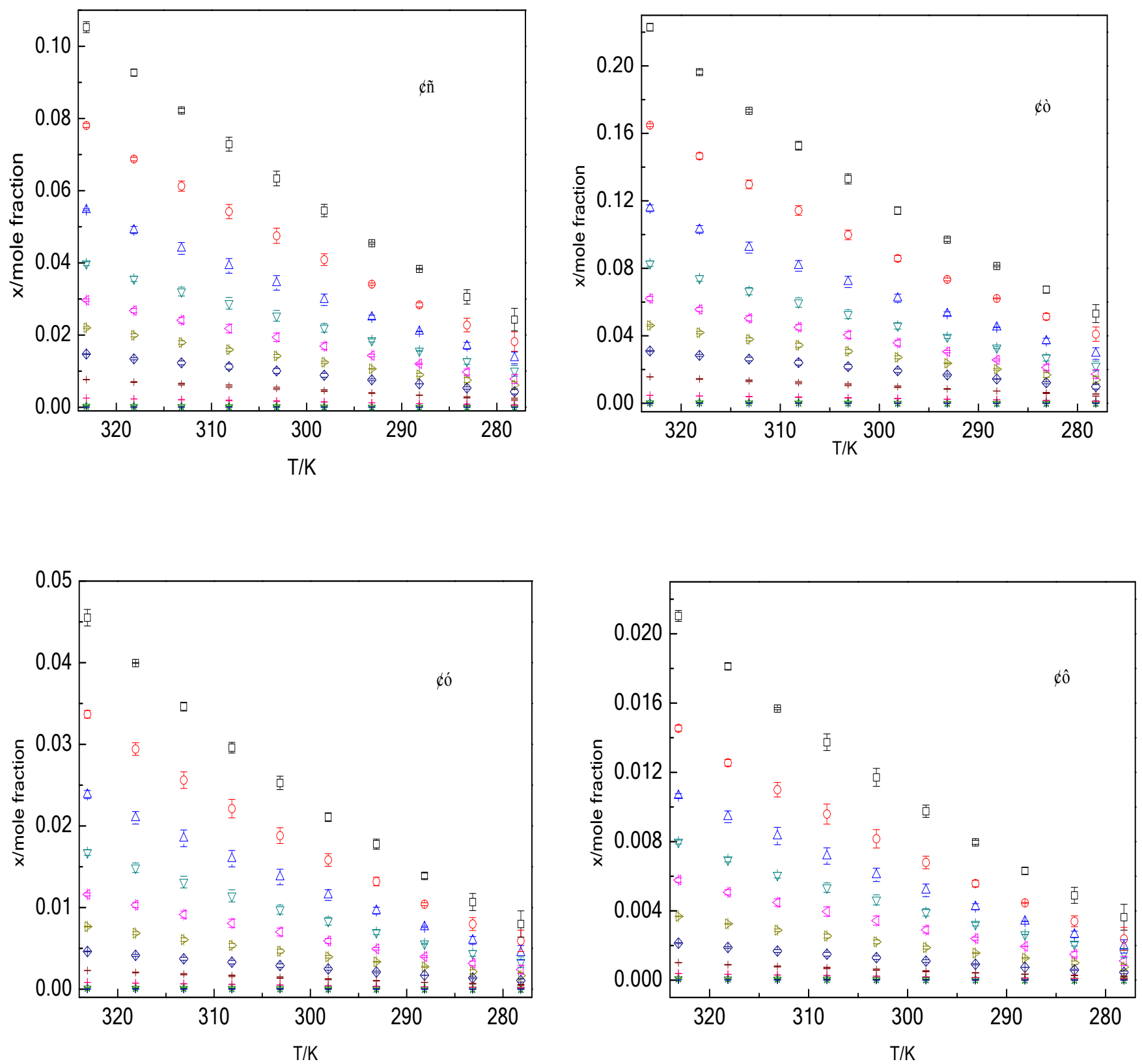

Fig S4. The deviation plot between moxidectin solubility (x) and calculated value of Van't Hoff-Jouyban-Acree model in four mixtures with various mass fractions at different temperatures: w, mass fraction of methanol ( I ); ethanol (II); isopropanol (III) and $\mathrm{EG}(\mathrm{IV}) ; \square, \mathrm{w}=1 ; \bigcirc, \mathrm{w}=0.9 ; \triangle, \mathrm{w}=0.8 ; \nabla, \mathrm{w}=0.7 ; \triangleleft, \mathrm{w}=0.6 ; \triangleright, \mathrm{w}=0.5 ; \diamond, \mathrm{w}=0.4 ; \square-, \mathrm{w}=0.3 ; \mid$, $\mathrm{w}=0.2 ;$ 访, $\mathrm{w}=0.1 ;+, \mathrm{w}=0$. 

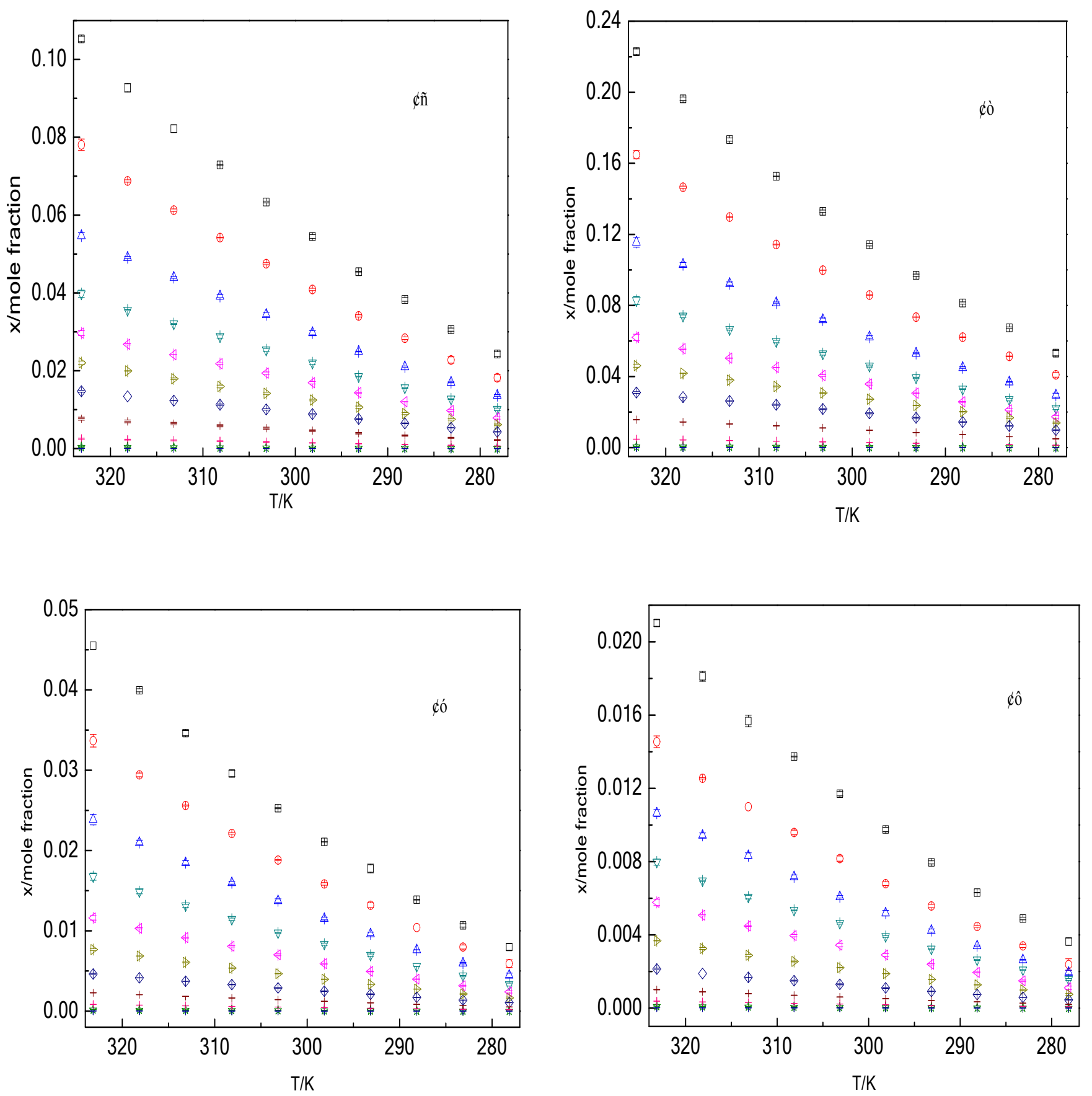

Fig S5. The deviation plot between moxidectin solubility (x) and calculated value of Modified Apelblat -Jouyban-Acree model in four mixtures with various mass fractions at different temperatures: w, mass fraction of methanol ( I ); ethanol (II); isopropanol (III) and EG (IV); $\square, \mathrm{w}=1 ; \bigcirc, \mathrm{w}=0.9 ; \triangle, \mathrm{w}=0.8 ; \nabla, \mathrm{w}=0.7 ; \triangleleft, \mathrm{w}=0.6 ; \triangleright, \mathrm{w}=0.5 ; \diamond, \mathrm{w}=0.4 ; \square-$ $\mathrm{w}=0.3 ; \mid, \mathrm{w}=0.2 ;$ 々, $\mathrm{w}=0.1 ;+, \mathrm{w}=0$. 


\section{Description of standard calculation model}

$$
\ln x_{\mathrm{w}, \mathrm{T}}=w_{1} \ln x_{1, \mathrm{~T}}+w_{2} \ln x_{2, \mathrm{~T}}+\frac{w_{1} w_{2}}{T / \mathrm{K}} \sum_{\mathrm{i}=0}^{2} J_{\mathrm{i}}\left(w_{1}-w_{2}\right)^{\mathrm{i}}
$$

Equation 1. Jouyban-Acree Model; $x_{\mathrm{w}, \mathrm{T}}$, solubility of solute at $T ; w_{1}$ and $w_{2}$, ratio of two solvents; $x_{1, \mathrm{~T}}$, and $x_{2, \mathrm{~T}}$, solubility in two pure solvents; $J_{\mathrm{i}}$ are parameters.

$$
\ln x_{\mathrm{T}}=A+\frac{B}{T / \mathrm{K}}
$$

Equation 2. Van't Hoff Model.

$$
\ln x_{\mathrm{w}, \mathrm{T}}=w_{1}\left(A_{1}+\frac{B_{1}}{T / \mathrm{K}}\right)+w_{2}\left(A_{2}+\frac{B_{2}}{T / \mathrm{K}}\right)+\frac{w_{1} w_{2}}{T / \mathrm{K}} \sum_{i=0}^{2} J_{i}\left(w_{1}-w_{2}\right)^{i}
$$

Equation 3. Van't Hoff-Jouyban-Acree Model; $A_{1}, B_{1}, A_{2}, B_{2}$ and $J_{\mathrm{i}}$ are model parameters.

$$
\ln x_{\mathrm{T}}=A+\frac{B}{T / K}+C \ln (T / K)
$$

Equation 4. Apelblat Model; $A, B$, and $C$ are parameters.

$$
\ln x_{\mathrm{w}, \mathrm{T}}=w_{1}\left[A_{1}+\frac{B_{1}}{T / \mathrm{K}}+C_{1} \ln (T / K)\right]+w_{2}\left[\left(A_{2}+\frac{B_{2}}{T / \mathrm{K}}+C_{2} \ln (T / K)\right]+\frac{w_{1} w_{2}}{T / \mathrm{K}} \sum_{i=0}^{2} J_{\mathrm{i}}\left(w_{1}-w_{2}\right)^{\mathrm{i}}\right.
$$

Equation 5. Modified Apelblat-Jouyban-Acree Model; $A_{1}, B_{1}, \mathrm{C}_{1}, A_{2}, B_{2}, \mathrm{C}_{2}$ and $J_{\mathrm{i}}$ are model parameters. 